DYNAMICAL

Sy ROMCTry 


\title{
DYNAMICAL
}

\section{Carl E Wulfman}

\author{
University of the Pacific, USA
}




\section{Published by}

World Scientific Publishing Co. Pte. Ltd.

5 Toh Tuck Link, Singapore 596224

USA office: 27 Warren Street, Suite 401-402, Hackensack, NJ 07601

UK office: 57 Shelton Street, Covent Garden, London WC2H 9HE

\section{British Library Cataloguing-in-Publication Data}

A catalogue record for this book is available from the British Library.

\section{DYNAMICAL SYMMETRY}

Copyright (C) 2011 by World Scientific Publishing Co. Pte. Ltd.

All rights reserved. This book, or parts thereof, may not be reproduced in any form or by any means, electronic or mechanical, including photocopying, recording or any information storage and retrieval system now known or to be invented, without written permission from the Publisher.

For photocopying of material in this volume, please pay a copying fee through the Copyright Clearance Center, Inc., 222 Rosewood Drive, Danvers, MA 01923, USA. In this case permission to photocopy is not required from the publisher.

ISBN-13 978-981-4291-36-1

ISBN-10 981-4291-36-6

Typeset by Stallion Press

Email: enquiries@stallionpress.com

Printed in Singapore. 


\section{Dedication}

To my fellow scientists who paused to encourage as they explored new vistas - Leonello Paoloni, John Platt, Marcos Moshinsky, Brian Judd, Brian Wybourne, Raphy Levine, and John Avery. 
This page is intentionally left blank 


\section{Preface}

In his Harmonices Mundi, published in 1619, Johannes Kepler exclaims, "Finally I have brought to light and verified beyond all my hopes and expectations that the whole Nature of Harmonies permeates to the fullest extent, and in all its details, the motion of heavenly bodies; not, it is true, in the manner in which I had earlier thought, but in a totally different, altogether complete way."

This book deals with concepts of symmetry which, nearly four centuries after Kepler, continue to provide ever deepening, and often surprising, understandings of interrelations between phenomena associated with moving bodies.

Kepler's religious convictions and his intuition led him to believe that geometric symmetries and musical harmonies are expressed in natural phenomena. The development of the calculus and Newtonian physics in the latter half of the seventeenth century made it possible to deduce and extend Kepler's three laws of planetary motion, but they were seldom seen as expressions of symmetries and harmonies in the natural world. Then, two and a half centuries after we were given Harmonices Mundi, our concepts of symmetry were profoundly deepened by Sophus Lie. Lie saw that any system governed by differential equations exhibits symmetries in a heretofore unrecognized sense. Because so many laws of nature can be formulated as differential equations, Lie's concept of symmetry makes it possible to establish a host of relationships between natural phenomena. By mid twentieth century, physicists had come to realize that relationships expressing Lie symmetries, though easily overlooked, are surprisingly useful. Now, much modern physics has become a search, first for symmetries, then, secondly, for the dynamics that produces them.

Readers of Lie's works cannot help but realize that much of his mathematics was guided by geometric intuitions. The extraordinary fertility 
of his thought processes, beyond a shadow of doubt, arose in part from the extraordinary visual capabilities of the human mind. Just as a picture can be "worth a thousand words", so also can a geometric illustration, even a non-Euclidean one, suggest a host of mathematical and physical relationships. For this reason, the first three chapters of this book use interconnected geometrical, analytic, and physical concepts of symmetry to stimulate questions as well as insights in the mind of the reader.

A more formal treatment of the subject begins in Chapter 4. Among other things, Chapters 4 and 6 establish the manner in which Lie symmetries are defined by differential equations. Chapter 5 explains how one determines the invariance transformations of functions, functionals, and equations. Readers may wish to shift back and forth between the first three chapters, and these chapters.

The remaining chapters of the book deal with physical and chemical consequences of the symmetries implied in the dynamical equations of mechanics, quantum mechanics, and electromagnetism. Invariance transformations of partial differential equations are discussed in Chapter 9, the first chapter dealing with Schrödinger equations.

A number of monographs currently in print deal with the invariance properties of differential equations, and several recent and very fine ones deal with the utilization of these properties in applied mathematics and engineering. A list of these will be found in the Bibliography at the end of this Preface. Helpful, but out of print, monographs are also listed.

The approach taken in this book contrasts with those to be found in the books of the Bibliography: here, geometric concepts play a greater role. It is hoped that the reader's natural geometric understanding of ordinary symmetry, will, like Lie's, be extended into a partly intuitive, geometric as well as analytic, understanding of symmetries not seen in the space of our common experience. A good deal of emphasis is placed upon dynamical symmetries of classical and quantum mechanical systems of interest to both chemists and physicists. Readers interested in engineering or applied mathematics may also find that the book provides viewpoints and analyses which can enrich their studies.

\section{References}

[1] G. W. Bluman, S. Kumei, Symmetries and Differential Equations (SpringerVerlag, N.Y., 1989).

[2] J. E. Campbell, Continuous Groups; reprint of the 1903 edition of Introductory Treatise on Lie's Theory of Finite Continuous Transformation Groups (Chelsea, Bronx, N.Y, 1966). 
[3] B. J. Cantwell, Introduction to Symmetry Analysis (Cambridge University Press, Cambridge, 2002).

[4] A. Cohen, The Lie Theory of One-Parameter Groups; reprint of the 1911 edition (Stechert, N.Y., 1931).

[5] N. H. Ibragimov, CRC Handbook of Lie Group Analysis of Differential Equations, Vol. 1, 1994-6 (CRC Press, N.Y. 1996).

[6] S. Lie, Theorie der Transformation Gruppen, I; translated by M. Ackerman, entitled Sophus Lie's 1880 Transformation Group Paper; comments by R. Hermann (Math Sci Press, Brookline, Mass, 1975).

[7] Peter J. Olver, Applications of Lie Groups to Differential Equations (Springer-Verlag, N.Y., 1986).

[8] J. M. Page, Ordinary differential Equations with an Introduction to Lie's Theory of the Group of One Parameter (Macmillan, London, 1897).

[9] H. Stephani, Differential Equations: Their Solution Using Symmetries (Cambridge University Press, Cambridge, 1989).

[10] P. Hydon, Symmetry Methods for Differential Equations (Cambridge University Press, Cambridge, 2000).

AUDIENCE: Primarily physicists, physical chemists, applied mathematicians and other natural scientists with a mathematical bent.

BACKGROUND ASSUMED: Differential and integral calculus, introduction to ordinary differential equations. The familiarity with classical mechanics and/or quantum mechanics attained by undergraduate students of physics by their junior or senior year, or by first-year graduate students of physical chemistry. 
This page is intentionally left blank 


\section{Acknowledgments}

It is a great pleasure to be able to acknowledge my indebtedness to those who have provided so much of the mental stimulation leading to these pages: to my parents Eugene and Jean, my wife Constance, and my brother, David; to some very special teachers, Christian Rondestvedt, George Uhlenbeck, and Michael Dewar; and to my students Fred Yarnell, Tai-ichi Shibuya, Sukeyuki Kumei, Yutaka Kitigawara, Yuuzi Takahata, and Gerald Hyatt.

Jerry Blakefield, Rochelle Wolber, and Jack Bench provided invaluable help checking and proofreading the manuscript, and Eve Wallis greatly improved many of the figures.

Generous grants from the Research Corporation, the Lindbergh Foundation, the University of the Pacific, and the Fulbright Commission, as well as grants from the National Science Foundation, supported research that was instrumental to the development of this book. 
This page is intentionally left blank 


\section{Contents}

Dedication $\quad$ v

Preface vii

Acknowledgements $\quad$ xi

1. Introduction 1

1.1 On Geometric Symmetry and Invariance in the Sciences 1

1.2 Fock's Discovery . . . . . . . . . . . . 6

1.3 Keplerian Symmetry . . . . . . . . . . . . . 8

1.4 Dynamical Symmetry . . . . . . . . . . 8

1.5 Dynamical Symmetries Responsible for Degeneracies and Their Physical Consequences . . . . . . . . . . . . . 10

1.6 Dynamical Symmetries When Energies Can Vary . . . . 12

1.7 The Need for Critical Reexamination of Concepts of Physical Symmetry. Lie's Discoveries . . . . . . . . . 13

Appendix A: Historical Note . . . . . . . . . . . . . . . . . . . 18

References .................. 19

2. Physical Symmetry and Geometrical Symmetry 23

2.1 Geometrical Interpretation of the Invariance Group of an Equation; Symmetry Groups . . . . . . . . . . 23

2.2 On Geometric Interpretations of Equations . . . . . . . 29

2.3 Geometric Interpretations of Some Transformations in the Euclidean Plane . . . . . . . . . . . . . . . . . . . 30

2.4 The Group of Linear Transformations of Two Variables 36 
2.5 Physical Interpretation of Rotations . . . . . . . . . . 37

$2.6 \quad$ Intrinsic Symmetry of an Equation . . . . . . . . . . . . 39

2.7 Non-Euclidean Geometries . . . . . . . . . . . . . . . . 40

2.8 Invariance Group of a Geometry . . . . . . . . . . . . . 48

2.9 Symmetry in Euclidean Spaces . . . . . . . . . . . . . . 49

2.10 Symmetry in the Spacetime of Special Relativity . . . . 51

2.11 Geometrical Interpretations of Nonlinear Transformations: Stereographic Projections . . . . . . . . . . 56

2.12 Continuous Groups that Leave Euclidean and PseudoEuclidean Metrics Invariant . . . . . . . . . . . . 62

2.13 Geometry, Symmetry, and Invariance . . . . . . . . . . 65

Appendix A: Stereographic Projection of Circles . . . . . . . 66

References ................... 68

\section{On Symmetries Associated With Hamiltonian} Dynamics

3.1 Invariance of a Differential Equation . . . . . . . . . 71

3.2 Hamilton's Equations . . . . . . . . . . . . . . 73

3.3 Transformations that Convert Hamilton's Equations into Hamilton's Equations; Symplectic Groups . . . . 76

3.4 Invariance Transformations of Hamilton's Equations of

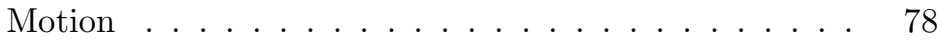

3.5 Geometrization of Hamiltonian Mechanics . . . . . . . 80

3.6 Symmetry in Two-dimensional Symplectic Space . . . . 91

3.7 Symmetry in Two-dimensional Hamiltonian Phase Space 92

3.8 Symmetries Defined by Linear Symplectic Transformations 95

3.9 Nonlinear Transformations in Two-dimensional Phase Space . . . . . . . . . . . . . . . 97

3.10 Dynamical Symmetries as Intrinsic Symmetries of

Differential Equations and as Geometric Symmetries . . 102

Exercises . . . . . . . . . . . . . . . . . . . 104

References ... . . . . . . . . . . . . . . . . 104

4. One-Parameter Transformation Groups 105

$4.1 \quad$ Introduction . . . . . . . . . . . . . . . . 105

4.2 Finite Transformations of a Continuous Group Define Infinitesimal Transformations and Vector Fields . . . . 108

4.3 Spaces in which Transformations will be Assumed to Act 112 
4.4 The Defining Equations of One-Parameter Groups of Infinitesimal Transformations. Group Generators ................. 114

4.5 The Differential Equations that Define Infinitesimal Transformations Define Finite Transformation Groups ................. . 116

4.6 The Operator of Finite Transformations . . . . . . . . . 119

4.7 Changing Variables in Group Generators . . . . . . . . 122

4.8 The Rectification Theorem . . . . . . . . . . . . . 124

4.9 Conversion of Non-autonomous ODEs to Autonomous ODEs .......................... 129

4.10 N-th Order ODEs as Sets of First-order ODEs . . . . . 131

4.11 Conclusion .................. 131

Appendix: Homeomorphisms, Diffeomorphisms, and Topology . . . . . . . . . . . . . . . . . . . 132

Exercises . . . . . . . . . . . . . . . . . 133

References .................. 134

5. Everywhere-Local Invariance

5.1 Invariance under the Action of One-Parameter Lie Transformation Groups . . . . . . . . . . . . . . . . 135

5.2 Transformation of Infinitesimal Displacements . . . . . 141

5.3 Transformations and Invariance of Work, Pfaffians, and Metrics . . . . . . . . . . . . . . . . 143

5.4 Point Transformations of Derivatives . . . . . . . . . . . 147

5.5 Contact Transformations . . . . . . . . . . . . . . 149

5.6 Invariance of an Ordinary Differential Equation of First-order under Point Transformations; Extended Generators .................. 151

5.7 Invariance of Second-order Ordinary Differential Equations under Point Transformations. Harmonic Oscillators ................. 154

5.8 The Commutator of Two Operators . . . . . . . . . . . 156

5.9 Invariance of Sets of ODEs. Constants of Motion . . . . 158

5.10 Conclusion ................. . 161

Appendix A. Relation between Symmetries and Integrating Factors .................. 162 
Appendix B. Proof That The Commutator of Lie Generators is Invariant Under Diffeomorphisms . . . . . . . . . . . 163

Appendix C. Isolating and Non-isolating Integrals of Motion . . . . . . . . . . . . . . 165

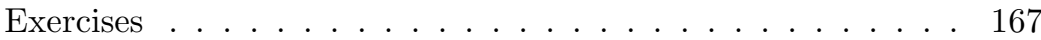

References ................... 167

6. Lie Transformation Groups and Lie Algebras 169

6.1 Relation of Many-Parameter Lie Transformation Groups to Lie Algebras . . . . . . . . . . . . . . . . . . . . . 169

6.2 The Differential Equations that Define Many-Parameter Groups . . . . . . . . . . . . . . . . 175

6.3 Real Lie Algebras . . . . . . . . . . . . . . . . 177

6.4 Relations between Commutation Relations and the Action of Transformation Groups: Some Examples . . . . . . . . . . . . . . . . . . 179

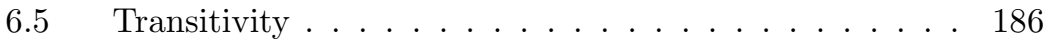

$6.6 \quad$ Complex Lie Algebras . . . . . . . . . . . . . . . . . 187

6.7 The Cartan-Killing Form; Labeling and Shift Operators . . . . . . . . . . . . . . 188

6.8 Casimir Operators . . . . . . . . . . . . . . . . . . . 191

6.9 Groups That Vary the Parameters of Transformation Groups . . . . . . . . . . . . . . . 192

6.10 Lie Symmetries Induced from Observations . . . . . . . 193

6.11 Conclusion . . . . . . . . . . . . . . . . . 195

Appendix A. Definition of Lie Groups by Partial Differential Equations . . . . . . . . . . . . . 196 Appendix B. Classification of Lie Algebras and Lie Groups . . . . . . . . . . . . . . . . 202 Exercises . . . . . . . . . . . . . . . . . . 204

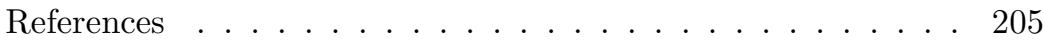

7. Dynamical Symmetry in Hamiltonian Mechanics 207

7.1 General Invariance Properties of Newtonian Mechanics . . . . . . . . . . . . . . . . . . . . . . . 207

7.2 Relationship of Phase Space to Abstract Symplectic Space . . . . . . . . . . . . . . . . . . . 210

7.3 Hamilton's Equations in PQ Space. Constants of Motion . . . . . . . . . . . . . . . . . . . . . . . . . 214 
7.4 Poisson Bracket Operators . . . . . . . . . . . . 217

7.5 Hamiltonian Dynamical Symmetries in PQ Space . . . 220

7.6 Hamilton's Equations in Classical PQET Space;

Conservation Laws Arising From Galilei Invariance . . . 230

7.7 Time-dependent Constants of Motion; Dynamical Groups That Act Transitively . . . . . . . . . . . . . . 234

7.8 The Symplectic Groups $\operatorname{Sp}(2 \mathrm{n}, \mathrm{r}) \ldots \ldots \ldots$

7.9 Generalizations of Symplectic Groups That Have an Infinite Number of One-Parameter Groups . . . . . . . 239

Appendix A. Lagranges Equation's and the Definition of Phase Space . . . . . . . . . . . . . . . . . . 240

Appendix B. The Variable Conjugate to Time in PQET Space . . . . . . . . . . . . . . . . 243

Exercises . . . . . . . . . . . . . . . . . 246

References ...................... 246

8. Symmetries of Classical Keplerian Motion 247

8.1 Newtonian Mechanics of Planetary Motion . . . . . . 247

8.2 Hamiltonian Formulation of Keplerian Motions in Phase Space . . . . . . . . . . . . . . 253

8.3 Symmetry Coordinates For Keplerian Motions . . . . . 257

8.4 Geometrical Symmetries of Bound Keplerian Systems in Phase Space . . . . . . . . . . . . . . . . . . 262

8.5 Symmetries of Keplerian Systems with Non-negative Energies . . . . . . . . . . . . . . 267

8.6 The $\mathrm{SO}(4,1)$ Dynamical Symmetry . . . . . . . . . 270

8.7 Concluding Remarks . . . . . . . . . . . . . . . 271

Exercises . . . . . . . . . . . . . . . . . . 272

References ..................... . . 273

9. Dynamical Symmetry in Schrödinger Quantum Mechanics

9.1 Superposition Invariance . . . . . . . . . . . . 275

9.2 The Correspondence Principle . . . . . . . . . . . 276

9.3 Correspondence Between Quantum Mechanical Operators and Functions of Classical Dynamical Variables . . . . . . . . . . . . . . . . . 280

9.4 Lie Algebraic Extension of the Correspondence Principle . . . . . . . . . . . . . . . . . . . . . 281 
9.5 Some Properties of Invariance Transformations of Partial Differential Equations Relevant to Quantum Mechanics . . . . . . . . . . . . . . 285

9.6 Determination of Generators and Lie Algebra of Invariance Transformations

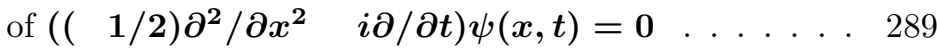

9.7 Eigenfunctions of the Constants of Motion of a Free-Particle . . . . . . . . . . . . . . . . . . . . . . 294

9.8 Dynamical Symmetries of the Schrödinger Equations of a Harmonic Oscillator . . . . . . . . . . 297

9.9 Use of the Oscillator Group in Pertubation Calculations . . . . . . . . . . . . . . . 300

9.10 Concluding Observations . . . . . . . . . . . . . 301

Historical Note . . . . . . . . . . . . . . . . . . . . . . . . . 302

Exercises . . . . . . . . . . . . . . . 302

References ................... . . 303

10. Spectrum-Generating Lie Algebras and Groups Admitted by Schröedinger Equations $\quad 305$

10.1 Lie Algebras That Generate Continuous Spectra . . . . 306

10.2 Lie Algebras That Generate Discrete Spectra . . . . . . 308

10.3 Dynamical Groups of N-Dimensional Harmonic Oscillators . . . . . . . . . . . . . . . . 309

10.4 Linearization of Energy Spectra by Time Dilatation; Spectrum-Generating Dynamical Group of Rigid Rotators . . . . . . . . . . . . . . . . 311

10.5 The Angular Momentum Shift Algebra; Dynamical Group of the Laplace Equation . . . . . . . . . . . . . 315

10.6 Dynamical Groups of Systems with Both Discrete and Continuous Spectra . . . . . . . . . . . . . 319

10.7 Dynamical Group of the Bound States of Morse Oscillators . . . . . . . . . . . . . . . . 320

10.8 Dynamical Group of the Bound States of Hydrogen-Like Atoms . . . . . . . . . . . . . . . . 322

10.9 Matrix Representations of Generators and Group Operators . . . . . . . . . . . . . 325

10.10 Invariant Scalar Products . . . . . . . . . . . . . . 327

10.11 Direct-Products: $\mathrm{SO}(3) \otimes \mathrm{SO}(3)$ and the Coupling of Angular Momenta . . . . . . . . . . . . . . . 328 
10.12 Degeneracy Groups of Non-interacting Systems.

Completions of Direct-Products . . . . . . . . . . . 329

10.13 Dynamical Groups of Time-dependent

Schrödinger Equations of Compound Systems.

Many-Electron Atoms . . . . . . . . . . . . . . . . . . . 331

Appendix A: References Providing Invariance Groups

of Schrödinger Equations . . . . . . . . . . . . . . . . . 334

Appendix B. Reference to Work Dealing with Dynamical

Symmetries in Nuclear Shell Theory . . . . . . . . . . . 334

Exercises ..................... 334

References ................... . . 335

\section{Dynamical Symmetry of Regularized} Hydrogen-like Atoms

11.1 Position-space Realization of the Dynamical

Symmetries . . . . . . . . . . . . . 337

11.2 The Momentum-space Representation . . . . . . . . . . 344

11.3 The Hyperspherical Harmonics $\mathrm{Y}_{\boldsymbol{k l m}}$. . . . . . . . . . . 349

11.4 Bases Provided by Eigenfunctions of $\mathrm{J}_{\mathbf{1 2}}, \mathrm{J}_{\mathbf{3 4}}, \mathrm{J}_{\mathbf{5 6}}$. . . 354

Appendix A. Matrix Elements of SO(4,2) Generators . . . . . 355

Appendix B. N-Shift Operators For the Hyperspherical

Harmonics . . . . . . . . . . . . . . 356

References .................. 359

12. Uncovering Approximate Dynamical Symmetries. Examples From Atomic and Molecular Physics 361

12.1 Introduction . . . . . . . . . . . . . . . . 361

12.2 The Stark Effect; One-Electron Diatomics . . . . . . . . 362

12.3 Correlation Diagrams and Level Crossings:

General Remarks . . . . . . . . . . . . . . . 364

12.4 Coupling $\mathrm{SO}(4)_{1} \otimes \mathrm{SO}(4)_{2}$ to Produce $\mathrm{SO}(4)_{12}$. . . . 369

12.5 Coupling $\mathrm{SO}(4)_{1} \otimes \mathrm{SO}(4)_{2}$ to Produce $\mathrm{SO}(4)_{1-2}$. . . . 371

12.6 Configuration Mixing in Doubly Excited States of Helium-like Atoms . . . . . . . . . . . . . . 373

12.7 Configuration Mixing Arising From Interactions

Within Valence Shells of Second and Third

Row Atoms . . . . . . . . . . . . . . . . . . 375

12.8 Origin of the Period-Doubling Displayed in Periodic Charts . . . . . . . . . . . . . . 375 
12.9 Molecular Orbitals in Momentum Space.

The Hyperspherical Basis . . . . . . . . . . . . . . . 377

12.10 The Sturmian Ansatz of Avery, Aquilanti and Goscinski . . . . . . . . . . . . . . . 380

Exercises . . . . . . . . . . . . . . . . . 384

References .................. . . 385

13. Rovibronic Systems 389

13.1 Introduction . . . . . . . . . . . . . . . 389

13.2 Algebraic Treatment of Anharmonic Oscillators With a Finite Number of Bound States . . . . . . . . . 389

$13.3 \mathrm{U}(2) \otimes \mathrm{U}(2)$ Model of Vibron Coupling . . . . . . . . . 394

13.4 Spectrum Generating Groups of Rigid Body Rotations . . . . . . . . . . . . . . . 396

13.5 The U(4) Vibron Model of Rotating Vibrating Diatomics . . . . . . . . . . . . 401

13.6 The $\mathrm{U}(4) \otimes \mathrm{U}(4)$ Model of Rotating Vibrating Triatomics . . . . . . . . . . . . 403

13.7 Concluding Remarks . . . . . . . . . . . . . 406

Exercises . . . . . . . . . . . . . . . 406

References .................. 406

14. Dynamical Symmetry of Maxwell's Equations 409

14.1 The Poincare Symmetry of Maxwell's Equations . . . . 410

14.2 The Conformal and Inversion Symmetries of Maxwell's Equations; Their Physical Interpretation . . . . . . . . . . . . . 413

14.3 Alteration of Wavelengths and Frequencies by a Special Conformal Transformation: Interpretation of Doppler Shifts in Stellar Spectra ................ . 420

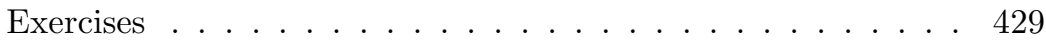

References .................. 431 